\title{
An assessment of 24-hour ambulatory EEG/ECG monitoring in a neurology clinic
}

\author{
RE CULL \\ From the Department of Medical Neurology, Royal Infirmary, Edinburgh, Scotland, UK
}

SUMMARY The relative merits of 24-hour ambulatory EEG/ECG monitoring and routine EEG recording have been compared in a group of 62 patients attending a neurological clinic because of episodes of loss of consciousness. Overall, ambulatory EEG abnormalities were detected in 21 cases $(34 \%)$ compared with 16 cases $(26 \%)$ for routine EEG. Ambulatory EEG mainly improved the detection of generalised paroxysmal activity, but in some cases lateralised abnormalities were detected which were not present on the routine record. Patients who reported clinical attacks once a week or more frequently showed an improved yield of diagnostic abnormalities on ambulatory EEG, but the technique did not prove superior to routine EEG if attacks were less frequent. The indications for, and limitations of, ambulatory EEG monitoring are discussed.

Over the past 8 years technical equipment has become available for the prolonged monitoring of EEG and ECG in ambulant patients, enabling a continuous 24-hour record of up to four data channels to be stored on a single magnetic tape cassette. ${ }^{2}$ The generalised spike-and-wave paroxysms of petit mal epilepsy are readily recorded by such systems, and the wave form associated with absence attacks can be detected by automatic systems which permit rapid replay and analysis of recorded tapes. ${ }^{3}$ This type of monitoring is of considerable value in the assessment of patients with absence seizures; the accuracy of the EEG record in assessing seizure frequency is considerably better than estimates provided by patients or their relatives. ${ }^{4}$ Although partial seizures and tonic-clonic attacks also can be monitored readily with an ambulatory system, the limited number of EEG channels available and the generally lower frequency of clinical attacks has tended to lead to selection of patients considered suitable for ambulatory monitoring. In the first few years after the introduction of ambulatory EEG/ ECG monitoring the major attraction of the system seemed to be the possibility of recording clinical attacks, thereby correlating clinical symptoms to neurophysiological abnormatities. For these reasons

Address for reprint requests: Dr RE Cull, Department of Medical Neurology, Royal Infirmary, Edinburgh EH3 9YW, UK.

Received 31 January 1984 and in final revised form 4 July 1984. Accepted 14 July 1984 patients have usually been selected because of frequent clinical symptoms in order that the chance of recording a clinical attack was relatively high. ${ }^{4}$ The present study was set up to see whether ambulatory EEG/ECG monitoring would provide useful diagnostic information in patients who were not selected because of frequent attacks but who had been referred to a neurological clinic because of episodes of loss of consciousness or altered awareness. The study was initiated to determine whether the routine use of ambulatory 24-hour EEG/ECG monitoring provided a better yield of diagnostic information compared with conventional EEG recording.

\section{Methods}

All the patients in this study were attending the medical neurology clinics in the Royal Infirmary, or Northern General Hospital, Edinburgh. They were referred because of episodes of loss of consciousness or impaired awareness which were felt by the referring physician possibly to be due to epilepsy. The majority of the cases were seen by one neurologist (REC) but a small number of cases were referred for ambulatory monitoring by other neurologists.

All patients had one or more routine 8-channel EEG records carried out using at least three standard montages, a period of hyperventilation and photic stimulation as routine procedures. The average duration of recording was $17 \mathrm{~min}$ (range 15-25 min). The EEG records were read by a clinical neurophysiologist. Ambulatory EEG/ECG 24hour monitoring was carried out using an Oxford Medilog 4-24 system employing two on-head pre-amplifiers for EEG, and one ECG channel. The fourth channel was used 
Tâble 1 Routine EEG

\begin{tabular}{lcc}
\hline & $n$ & $(\%)$ \\
\hline Normal & 35 & $(56)$ \\
Equivocal & 11 & $(18)$ \\
Focal abnormality & 7 & $(11)$ \\
Generalised abnormality & 9 & $(15)$ \\
\hline
\end{tabular}

Table 2 Ambulatory EEG/ECG

\begin{tabular}{lrr}
\hline & $n$ & $(\%)$ \\
\hline Normal EEG & 37 & $(60)$ \\
Equivocal EEG & 4 & $(6)$ \\
Focal EEG abnormality & 8 & $(13)$ \\
Generalised EEG abnormality & 13 & $(21)$ \\
Equivocal ECG & 5 & $(8)$ \\
Abnormal ECG & 0 & $(0)$ \\
\hline
\end{tabular}

for a digital time code to allow accurate timing of clinical events. In most cases a mid-parietal montage $\left(\mathrm{C}_{4}-\mathrm{P}_{4}\right.$, $\mathrm{C}_{3}-\mathrm{P}_{3}$ ) was used but in some instances a mid-temporal montage was employed. The patient was asked to record a list of daily events and clinical symptoms on a diary sheet, and an event button was available on the recorder so that clinical attacks could be registered on the tape by a marking signal. Most of the patients were allowed home after the recording equipment had been fitted, and returned the next day for it to be removed. In a few instances patients being investigated in hospital were recorded in the hospital wards. Playback of the EEG/ECG data was carried out using an Oxford Medical Systems PMD-12 page-mode display system which permits recordings to be reviewed at up to 60 times real time. The records were reviewed by a single observer (REC).

\section{Results}

\section{Patients}

Sixty-two patients were recorded (27 males, 35 females). Their age range was 13-81 years (mean age $35 \cdot 3$ years). Ambulatory 24-hour EEG/ECG monitoring was carried out on 70 occasions, records being repeated on eight patients because of technical failure (three cases), equivocal results (three cases) or failure to record attacks in patients with otherwise frequent symptoms (two cases).

\section{Clinical problems}

The neurologists referring patients for ambulatory
Table 4

\begin{tabular}{lll}
\hline & Routine EEG & Ambulatory EEG \\
\hline Normal/equivocal & $46(74 \%)$ & $41(66 \%)$ \\
Abnormal & $16(26 \%)$ & $21(34 \%)$ \\
Total & 62 & 62 \\
\hline
\end{tabular}

monitoring felt that the diagnosis was likely to be epilepsy in 32 cases $(52 \%)$, syncope in 11 cases $(18 \%)$ and psychological in four cases $(6 \%)$. The clinical assessment was quite uncertain in 15 cases (24\%).

\section{Results of routine EEG recording}

Results are shown in table 1 . Routine EEG ( $R$ EEG) was considered normal or equivocal in 46 cases $(74 \%)$. The records showed a focal abnormality in seven cases $(11 \%)$ and generalised abnormalities in nine cases (15\%). When R/EEG was abnormal (16 cases) this was due to focal slow waves in three cases (19\%), focal spikes or sharp waves in four cases $(25 \%)$, generalised spike-and-wave paroxysms in eight cases $(50 \%)$ or generalised slow wave abnormalities in one case $(6 \%)$.

Ambulatory EEG/ECG (table 2)

The ambulatory EEG (A/EEG) record was felt to be normal or equivocal in 41 cases $(66 \%)$. A lateralised abnormality was detected in eight cases $(13 \%)$ and generalised EEG abnormalities in 13 cases (21\%). The ECG record was felt to be equivocal in five cases $(8 \%)$ and in these only minor degrees of tachycardia or bradycardia were noted (table 3). No case was seen where abnormalities of the ECG likely to cause loss of consciousness were detected. When the A/EEG record was abnormal (21 cases) this was due to focal slow waves in two cases $(10 \%)$, focal spikes or sharp waves in six cases $(28 \%)$ and generalised spike and wave discharges in 13 cases (62\%).

An overall comparison of abnormalities detected with $R / E E G$ and $A / E E G$ is shown in table 4 . It can be seen that in the total group of 62 patients R/EEG recording was abnormal in 16 cases $(26 \%)$ while $\mathrm{A} / \mathrm{EEG}$ recording was abnormal in 21 cases $(34 \%)$. This difference does not reach statistical significance

Table 3 Summary of ECG abnormalities

\begin{tabular}{llll}
\hline Patient & Sex & Age $(y r)$ & ECG \\
\hline 1 & F & 77 & Recurrent single ventricular ectopic beats. \\
& Sinus rhythm 60-70/min. \\
2 & M & 20 & Recurrent single ventricular and supraventricular ectopic beats. \\
3 & F & 81 & Sinus tachycardia up to $160 / \mathrm{min}$. \\
4 & M & 36 & Sinus tachycardia up to $170 / \mathrm{min}$. \\
5 & 52 & Sinus tachycardia up to $160 / \mathrm{min}$.
\end{tabular}

Five out of 62 patients had equivocal ECG. 
Table 5

\begin{tabular}{|c|c|c|c|c|c|}
\hline \multirow[t]{2}{*}{ Routine EEG } & & \multicolumn{4}{|c|}{ Ambulatory EEG } \\
\hline & & Normal & Equivocal & Focal $A B N$ & $\overline{\text { Generalised } A B N}$ \\
\hline $\begin{array}{l}\text { Normal } \\
\text { Equivocal } \\
\text { Focal ABN } \\
\text { Generalised ABN }\end{array}$ & $\begin{array}{r}35 \\
11 \\
7 \\
9\end{array}$ & $\begin{array}{r}30 \\
5 \\
1 \\
1\end{array}$ & $\begin{array}{l}2 \\
2 \\
0 \\
0\end{array}$ & $\begin{array}{l}1 \\
2 \\
4 \\
1\end{array}$ & $\begin{array}{l}2 \\
2 \\
2 \\
7\end{array}$ \\
\hline
\end{tabular}

using McNemar's test $(0 \cdot 1<\mathrm{p}<0 \cdot 2)$.

Table 5 shows a comparison of the two recording techniques in various sub-groups of the patients studied. It can be seen that of the 35 patients with normal R/EEG records, a lateralised EEG abnormality was detected with the ambulatory system in one case and generalised abnormalities in a further two. Of 11 patients with equivocal R/EEG records, ambulatory recording detected lateralised abnormalities in two and generalised abnormalities in a further two cases. Where R/EEG showed focal abnormalities in seven patients, A/EEG detected lateralised abnormalities in four cases and generalised abnormalities in two. Where generalised abnormalities were found on R/EEG recording in nine patients, the ambulatory system detected generalised abnormalities in seven and a lateralised abnormality in one case. Overall, therefore, out of 46 patients with normal or equivocal R/EEG records seven were found to have abnormal A/EEG recordings. Out of 16 cases with an abnormal $R /$ EEG, ambulatory recording failed to detect any abnormality in two cases $(12 \cdot 5 \%)$.

\section{Effect of clinical attack frequency}

When all 62 patients are grouped according to their reported clinical attack frequency (table 6) it can be seen that patients with attacks occurring monthly or less frequently were just as likely to have abnormalities on R/EEG as on 24-hour ambulatory records. However, two patients $(18 \%)$ with attacks occurring weekly had abnormalities on R/EEG compared with five cases $(45 \%)$ when $A / E E G$ recording was used. In patients with attacks occurring daily, R/EEG was abnormal in nine cases (43\%) while A/EEG showed abnormalities in 12 cases $(57 \%)$. When all patients with attacks occur-

Table 6 Effect of clinical attack frequency (62 patients)

\begin{tabular}{llc}
\hline Attack frequency & \multicolumn{2}{l}{$\begin{array}{l}\text { Numbers of abnormal EEG records } \\
\text { (percentage abnormal in brackets) }\end{array}$} \\
\cline { 2 - 3 } & R/EEG & A/EEG \\
\hline$<$ Monthly & $3(17)$ & $3(17)$ \\
Monthly & $2(17)$ & $1(8)$ \\
Weekly & $2(18)$ & $5(45)$ \\
Daily & $9(43)$ & $12(57)$ \\
\hline
\end{tabular}

ring once a week or more often are grouped together (32 cases), 11 (34\%) had abnormalities on R/EEG while A/EEG was abnormal in 17 cases (53\%). This difference just fails to reach statistical significance on McNemar's test $(0.05<p<0.1)$.

\section{Patients suspected of having psychologically determined attacks}

Four patients had attacks which were considered by the referring clinician likely to be psychologically determined. Two of these patients were male (ages 16 and 55), and two female (ages 16 and 26). Their reported attack frequency was high, being 1-8 attacks per day. In three of the four cases, clinical attacks occurred during $A / E E G$ recording and in all cases no EEG abnormalities were detected.

\section{Discussion}

This study has indicated that 24-hour ambulatory EEG/ECG monitoring can increase the detection of diagnostic abnormalities in patients being investigated for possible epilepsy when compared with routine EEG recordings. The results are in general agreement with those of Ebersole and Leroy ${ }^{5}$ who found A/EEG abnormalities in $37 \%$ of epileptic patients with normal R/EEG records. Although the number of EEG channels available for ambulatory recording on the current system is very limited, the greatly increased length of time of the EEG record compensates for this to a certain degree. It is of note that the major improvement in detection of paroxysmal abnormalities using ambulatory recording was in those cases where generalised disturbances were registered. A/EEG appears to be $95-100 \%$ efficient in the detection of generalised abnormalities, but may fail to detect $20 \%$ of focal temporal activity. ${ }^{5}$ Only a small increase in lateralised abnormalities was found with ambulatory recording in the present series, but in two of these cases very useful information was obtained as to the side of origin of the patient's complex partial seizures. A/EEG recording failed to detect localised abnormalities which had been detected on routine recording in two cases out of 16; a failure rate comparable to that of Ebersole and Leroy ${ }^{5}$ who found A/EEG missed $15 \%$ of abnormalities seen on simultaneous telemetered 
8-channel EEG. Therefore, ambulatory recording must be regarded as complementary to routine multi-channel recording rather than superior to it. As multi-channel ambulatory recording systems become more widely available this point may become less important.

The current study suggests that useful diagnostic information may be obtained from ambulatory EEG recording even if the likelihood of a clinical attack occurring is relatively low. Useful interictal abnormalities may be detected during wakefulness or sleep, but it will be noted that the ambulatory system was found superior to routine recording only in those patients who reported clinical attacks occurring once a week or more frequently. Although ambulatory EEG recording is relatively cheap in terms of material and technical time, it is more costly than routine recording because the record must be reviewed by an experienced interpreter and this process may take between 30-60 minutes depending on the complexity of the results.

Movement and muscle artefacts are frequent on A/EEG recordings and because of this the interpreter often has to disregard abnormalities which he thinks might be paroxysmal activity but are associated with other evidence of body movement or muscle activity. Although it is accepted that anterior temporal electrode montages detect more lateralised abnormalities, than do parietal positions ${ }^{6}$ it is unfortunate that the temporal montage often carries severe eye blink and temporalis muscle artefacts. The clinician must therefore weigh up the possible benefits of using a temporal montage against the greater ease of interpretation of records in which electrodes are sited away from the eyes or temporalis muscles.

The low incidence of ECG abnormalities in this group of patients is interesting. It has been claimed that up to $20 \%$ of patients attending clinics with the possible diagnosis of epilepsy may have cardiac arrhythmias sufficient to account for their attacks. In the group studied here, only minor ECG abnormalities were detected and in no case was this felt to indicate that there was a cardiac cause for their attacks. It may be that the previous estimate of cardiac causes of collapse by Schott et al ${ }^{7}$ it too high.
The age range in their study was $13-70$ years, and the average age of cases with disorders of cardiac rhythm 32.9 years. These figures are very similar to those of patients in the present study, and it is unlikely that the difference in detection of cardiac dysrhythmias is due to an age-dependent effect. It remains possible that differences in observers' criteria for "significant" cardiac rhythm abnormalities account for this discrepancy.

The author is grateful to $\mathrm{Mr} \mathrm{P}$ Wainwright for technical assistance, to Dr A McInnes for help with EEG reporting, to Dr EH Jellinek and Dr B Ashworth for referring patients, and to Dr R Prescott for statistical advice. Financial assistance from the Epilepsy Society of Edinburgh and South East Scotland for the purchase of ambulatory recording equipment is gratefully acknowledged.

\section{References}

' Quy RJ, Willison RG, Fitch P, Gilliatt RW. Some developments in ambulatory monitoring of the EEG In: Stott FD, Raftery EG, Sleight P, Gouding L, eds Proceedings of the Third International Symposium on

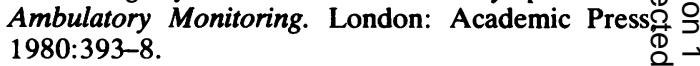

${ }^{2}$ Quy RJ. A miniature preamplifier for ambulatoryo 7 monitoring of the electroencephalogram. $J$ Physio (Lond) 1978; 234:23-4.

${ }^{3}$ Quy RJ, Fitch P, Willison RG. High-speech automatie analysis of EEG spike and wave activity using ananalogue detection and microcomputer plotting sys? tem. Electroencephologr Clin Neurophysiol 1980; or 49:187-9.

${ }^{4}$ Cull RE. Ambulatory EEG monitoring. In: Rose FC, ed. Research Progress in Epilepsy. London: Pitman 1983:269-72.

${ }^{5}$ Ebersole JS, Leroy RF. An evaulation of ambulatory, cassette EEG monitoring: II detection of interictal abnormalities. Neurology (NY) 1983;33:8-18.

${ }^{6}$ Leroy RF, Ebersole JS. An evaluation of ambulatory, cassette EEG monitoring: 1 Montage design. Neurology $(N Y)$ 1983;33:1-7.

' Schott GD, McLeod AA, Jewitt DE. Cardiac arrhythmias that masquerade as epilepsy. $\mathrm{Br}$ Med $J$ 1977;1:1454-7. 